\title{
Localized quench in $1+1$ conformal field theory
}

\author{
Maria Tikhanovskaya ${ }^{1, \star}$ \\ ${ }^{1}$ Steklov Mathematical Institute, Russian Academy of Sciences, Gubkin str. 8, 119991 Moscow, Russia
}

\begin{abstract}
We study the boundary dual of $\mathrm{AdS}_{3}$ spacetime with a point particle. Particles in $\mathrm{AdS}_{3}$ generate topological defects, which allows to formulate the geodesic image method for boundary correlators. We propose the generalization of the geodesic recipe to arbitrary time intervals in case of the bulk spacetime deformed by a point particle.
\end{abstract}

\section{Introduction}

The AdS/CFT - correspondence [1] is the correspondence between $(d+1)$ dimensional gravity of the AdS spacetime and $d$ - dimensional quantum field theory on the boundary of the space. The useful and important tool in holographic calculations is the geodesic approximation [2]. This approximation allows to get in a simple way the correlation function.

The geodesic approximation directly relates correlation functions of the boundary QFT to geometry of the bulk spacetime. However, the geodesic prescription is valid only either for Euclidean spacetimes, or for spacelike-separated points in the Lorentzian case. Timelike geodesics in asymptotically AdS spacetimes cannot reach the boundary, therefore the timelike region is unavailable to the prescription, unless there is an analytical continuation from the original Euclidean form. Because of this, the information carried by large-time dynamics, or even real-time correlators in general, cannot be obtained from the geodesics approximation in general spacetimes. In [3] a non-trivial Euclidean continuation was constructed for the Vaidya spacetime. Another method that was used in $[3,4]$ is making use of discontinuous timelike geodesics which go through Poincare horizon.

In our work the prescription for calculation two point correlation function in geodesic approximation for the case when the particle deforms the $\mathrm{AdS}_{3}$ spacetime is presented. Moving particle in the bulk realizes a quantum quench in the boundary theory. Here the formula for correlation function in geodesic approximation has the form:

$$
G_{\Delta}(a, b)=\sum e^{-\Delta \mathcal{L}_{r e n}(a, b)}
$$

We sum over all geodesics that connect $a$ and $b$ points. In the work [5] it was shown that geodesic approximation gives the exact answer (i.e. coincides with the answer from the $[6,7]$ ) for the correlation function if the $\mathrm{AdS}_{3}$ spacetime is orbifold and does not reproduce exact answer otherwise. We generalize the prescription to timelike-separated points by utilizing a set of auxiliary geodesics.

^e-mail: tikhanovskaya@mi.ras.ru 


\section{$2 \mathrm{AdS}_{3}$ spacetime with particles}

The $\mathrm{AdS}_{3}$ is maximally symmetric solution of Einstein equations with the negative cosmological constant. In the embedding coordinates the AdS spacetime is a hyperboloid. If we parametrize the embedding coordinates by global coordinates $(t, \chi, \phi)$ the metric of $\mathrm{AdS}_{3}$ spacetime can be written as follows:

$$
d s^{2}=-\cosh ^{2} \chi d t^{2}+d \chi^{2}+\sinh ^{2} \chi d \phi^{2}, \quad \phi \in(0,2 \pi)
$$

where $t$ is the time coordinate, $\chi$ is the radial coordinate and $\phi$ is the angular coordinate with period $2 \pi$. The $\mathrm{AdS}_{3}$ conformal boundary corresponds to $\chi \rightarrow \infty$.

\section{1 $\mathrm{AdS}_{3}$ spacetime with static particle}

We briefly discuss the Deser-Jackiw solution of Einstein equations with delta function in the RHS and cosmological constant equals to $-1[8,9]$. It describes a static particle in the center of the $\mathrm{AdS}_{3}$ spacetime. Solution of these equations has the form:

$$
d s^{2}=-\cosh ^{2} \chi d t^{2}+d \chi^{2}+\sinh ^{2} \chi d \phi^{2}, \quad \phi \in(0,2 \pi A) .
$$

The metric is the same as the metric for empty spacetime but an angle of the space has different ranges. Here $A$ is the parameter associated with the mass parameter:

$$
A=1-4 G \mu \in(0,1)
$$

where $G$ is the Newtonian constant and $\mu$ is the mass of the particle. In the Fig.1A we show the point particle in the center of the $\mathrm{AdS}_{3}$ spacetime. This particle cuts out the wedge and faces of the wedge must be glued.
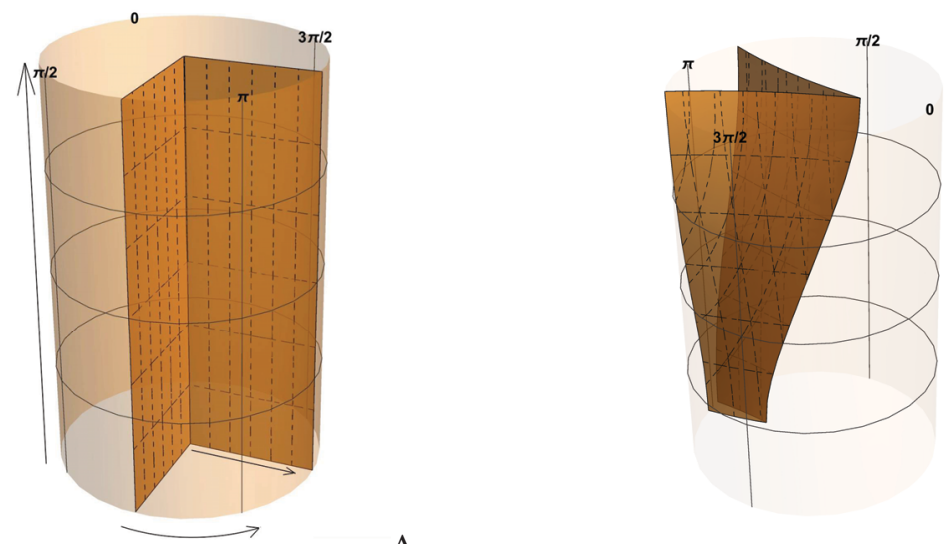

A.

B.

Figure 1. A. $\mathrm{AdS}_{3}$ with static massive particle in the center. Particle cats out the wedge from the spacetime. B. $\mathrm{AdS}_{3}$ with moving massive particle. Here the mass parameter $\alpha=\pi / 8$ and the boost parameter $\xi=1$. 


\section{2 $\mathrm{AdS}_{3}$ spacetime with moving particle}

In this section we explain how the moving particle can be obtained in the $\mathrm{AdS}_{3}$ spacetime. In [10] it was shown that using the group language for description the $\mathrm{AdS}_{3}$ spacetime with a particle we can boost this particle by Lorentz transformation and get the spacetime with a moving particle in a simple way. The particle moves periodically with the $T=2 \pi$ period. Massive particle does not reach the boundary.

Consider the action of the isometry in the $\mathrm{AdS}_{3}$ spacetime. The $*$-transformation is the isometry corresponding to the identification of the faces of the wedge. In the case of moving particle the isometry transformation is as follows [10]:

$$
\begin{aligned}
\tan t^{*} & =\mathcal{B}_{\xi}(\alpha) \sec t \tanh \chi \cos \phi+\tan t\left(1+2 \sinh ^{2} \xi \sin ^{2} \frac{\alpha}{2}\right) \\
\tan \phi^{*} & =-\frac{2 \tan \phi}{\mathcal{F}_{\xi}(\alpha)} \\
\cosh \chi^{*} & =\cosh \chi\left[\left(\mathcal{B}_{\xi}(\alpha) \tanh \chi \cos \phi+\sin t\left(1+2 \sinh ^{2} \xi \sin ^{2} \frac{\alpha}{2}\right)\right)^{2}+\cos ^{2} t\right]^{\frac{1}{2}} .
\end{aligned}
$$

where $\mathcal{B}_{\xi}$ and $\mathcal{F}_{\xi}$ are defined as

$$
\begin{aligned}
\mathcal{B}_{\xi}(\alpha) & =\sinh \xi\left(\sin \alpha \tan \phi-2 \cosh \xi \sin ^{2} \frac{\alpha}{2}\right), \\
\mathcal{F}_{\xi}(\alpha) & =\cosh \xi(2 \sin \alpha \tan \phi-\cos \alpha+\cos \phi)+\sec \phi \cos (\alpha+\phi) \\
& +\cos \alpha \cosh 2 \xi-2 \sinh ^{2} \xi .
\end{aligned}
$$

We are interested in the boundary theory so in the formulae (5) we take a limit of radial coordinate (i.e. $\chi \rightarrow \infty$ ) and get the action of isometry on the boundary ( $b$ means the boundary):

$$
\begin{aligned}
\tan t_{b}^{*} & =\mathcal{B}_{\xi}(\alpha) \sec t \cos \phi+\tan t\left(1+2 \sinh ^{2} \xi \sin ^{2} \frac{\alpha}{2}\right), \\
\tan \phi_{b}^{*} & =-\frac{2 \tan \phi}{\mathcal{F}_{\xi}(\alpha)} .
\end{aligned}
$$

In the Fig.1B we illustrate the $\mathrm{AdS}_{3}$ spacetime with a moving massive particle. With the expression for the induced boundary identification at hand, one can now formulate the geodesic images prescription for spacelike geodesics, which was explained in [10].

\section{Holographic image method and correlation function}

Consider the case of the empty $\mathrm{AdS}_{3}$ spacetime. For the calculation of two point correlation function we use the geodesics approximation [2]. In this approximation one defines the correlator:

$$
G_{\Delta}\left(\phi_{a}, t_{a} ; \phi_{b}, t_{b}\right)=e^{-\Delta \mathcal{L}_{r e n}\left(\phi_{a}, t_{a} ; \phi_{b}, t_{b}\right)},
$$

where $a$ and $b$ are boundary points with coordinates $\left(\phi_{a}, t_{a}\right)$ and $\left(\phi_{b}, t_{b}\right)$. If the interval between $a$ and $b$ points is spacelike then the $\mathcal{L}_{r e n}$ is the renormalized length of the geodesic connecting these points [10]. In case of timelike interval between $a$ and $b$ points we have to use the the prescription from the work [11].

The length of geodesic between two points in the $\mathrm{AdS}_{3}$ is:

$$
\mathcal{L}(a, b)=\ln \left[2\left(\cos \left(t_{a}-t_{b}\right)-\cos \left(\phi_{a}-\phi_{b}\right)\right)\right]
$$


then the correlation function (10) has the form:

$$
G_{\Delta, A d S}\left(\phi_{a}, t_{a} ; \phi_{b}, t_{b}\right)=\left(\frac{1}{2\left(\cos \left(t_{a}-t_{b}\right)-\cos \left(\phi_{a}-\phi_{b}\right)\right)}\right)^{\Delta} .
$$

In case of spacetime deformed by particle we have to change the general formulae for correlation function (12) and use the follows:

$$
\begin{aligned}
& G_{\Delta, l . s .}\left(\phi_{a}, t_{a} ; \phi_{b}, t_{b}\right)=\Phi_{\Delta}\left(\phi_{a}, t_{a} ; \phi_{b}, t_{b}\right) G_{\Delta, \mathrm{AdS}}\left(\phi_{a}, t_{a} ; \phi_{b}, t_{b}\right) \Theta_{0}\left(\phi_{a}, t_{a} ; \phi_{b}, t_{b}\right)+ \\
& +\sum_{n=1}^{n_{\max }} \Phi_{\Delta}\left(\phi_{a, n}^{*}, t_{a, n}^{*} ; \phi_{b}, t_{b}\right) G_{\Delta, \mathrm{AdS}}\left(\phi_{a, n}^{*}, t_{a, n}^{*} ; \phi_{b}, t_{b}\right) \Theta_{0}\left(\phi_{a, n}^{*}, t_{a, n}^{*} ; \phi_{b}, t_{b}\right) Z_{n}\left(\phi_{a, n}^{*}, t_{a, n}^{*} ; \phi_{b}, t_{b}\right)+ \\
& +\sum_{n=1}^{\bar{n}_{\max }} \Phi_{\Delta}\left(\phi_{a, n}^{\#}, t_{a, n}^{\#} ; \phi_{b}, t_{b}\right) G_{\Delta, \mathrm{AdS}}\left(\phi_{a, n}^{\#}, t_{a, n}^{\#} ; \phi_{b}, t_{b}\right) \bar{\Theta}_{0}\left(\phi_{a, n}^{\#}, t_{a, n}^{\#} ; \phi_{b}, t_{b}\right) \bar{Z}_{n}\left(\phi_{a, n}^{\#}, t_{a, n}^{\#} ; \phi_{b}, t_{b}\right),
\end{aligned}
$$

where $G_{\Delta, \text { AdS }}(\phi, t)$ is given by (12). The presence of the $\Phi$-factors in summands is related to the change of the causal relation between two points on the boundary under the isometry transformation (9).

Here the subscript 1.s. in the LHS means a living space of the boundary of the $\mathrm{AdS}_{3}$ with static or moving defects and

$$
\left(\phi_{a}, t_{a}\right)^{* n}=\left(\phi_{a, n}^{*}, t_{a, n}^{*}\right), \quad\left(\phi_{a}, t_{a}\right)^{\# n}=\left(\phi_{a, n}^{\#}, t_{a, n}^{\#}\right)
$$

are coordinates of the image points obtained by $n$-times applications of the isometry $*$ and \#transformations respectively. We can use the \#-transformation as an inverse $*$-transformation as follows:

$$
\left(\phi_{a}^{\#}, t_{a}^{\#}\right)^{*}=\left(\phi_{a}, t_{a}\right)
$$

In case of deformed AdS spacetime for calculation of correlation function we have to use the extra factors:

$$
\begin{array}{ll}
\Theta_{0}\left(\phi_{a, n}^{*}, t_{a, n}^{*} ; \phi_{b}, t_{b}\right), & \bar{\Theta}_{0}\left(\phi_{a, n}^{\#}, t_{a, n}^{\#} ; \phi_{b}, t_{b}\right), \\
Z_{\Delta, n}\left(\phi_{a, n}^{*}, t_{a, n}^{*} ; \phi_{b}, t_{b}\right), & \bar{Z}_{\Delta, n}\left(\phi_{a, n}^{\#}, t_{a, n}^{\#} ; \phi_{b}, t_{b}\right),
\end{array}
$$

where

$$
\Theta_{0}\left(\phi_{a}, t_{a}, \phi_{b}, t_{b}\right)= \begin{cases}1, & \left|\phi_{a}-\phi_{b}\right|>\left|t_{a}-t_{b}\right|, \\ 0, & \left|\phi_{a}-\phi_{b}\right|<\left|t_{a}-t_{b}\right|,\end{cases}
$$

Factors $Z$ are related to renormalizations [10].

Geodesic in (13) connecting points $a^{*}, a^{* *}, \ldots$ and $b$ are image geodesics. Length of each image geodesic equals to the length of a winding geodesic between $a$ and $b$ points [10].

The images prescription for the correlator can be generalized to the case when the bulk spacetime is deformed by several particles.

\section{Conclusion}

We have calculated the two point correlation function in holographic approach via geodesic approximation. We have considered the case when geometry of $\mathrm{AdS}_{3}$ is deformed by moving and static point massive particles. The whole Virasoro symmetry remains unchanged only in case when the mass parameter equals to $2 \pi / n$, where $n$ is integer (see Fig.2) but in the general case the symmetry is broken by the conical defect and this is manifest in the fact that correlation function in the geodesic approximation has the singularities in $\varphi$ dependence (see Fig.3). 

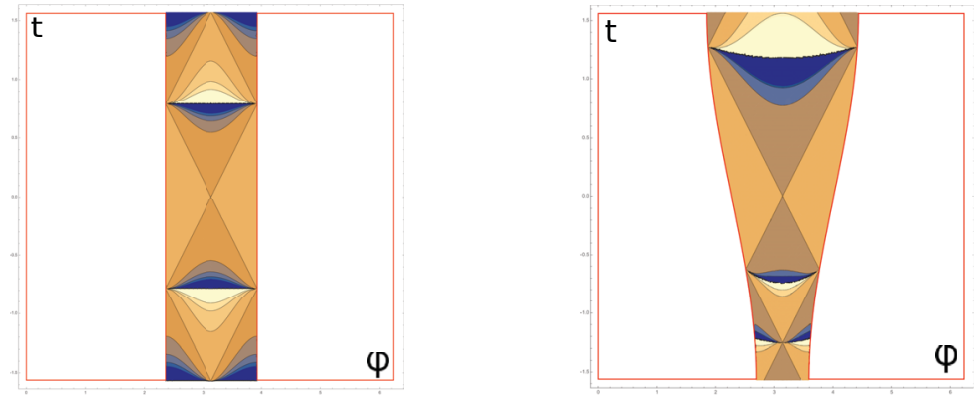

Figure 2. Density plot of inverse correlation function as a function of $G^{-1}(t, \varphi)$ is presented. In both of the cases the massive moving particle with the mass parameter $2 \pi / n$ where $n$ is integer deforms the $\mathrm{AdS}_{3}$ spacetime. Parameter values are: $\xi=0, \alpha=3 \pi / 2$. In the left plot the massive particle is static. In the right plot the massive particle is moving. Parameter values are: $\xi=0.6, \alpha=3 \pi / 2$
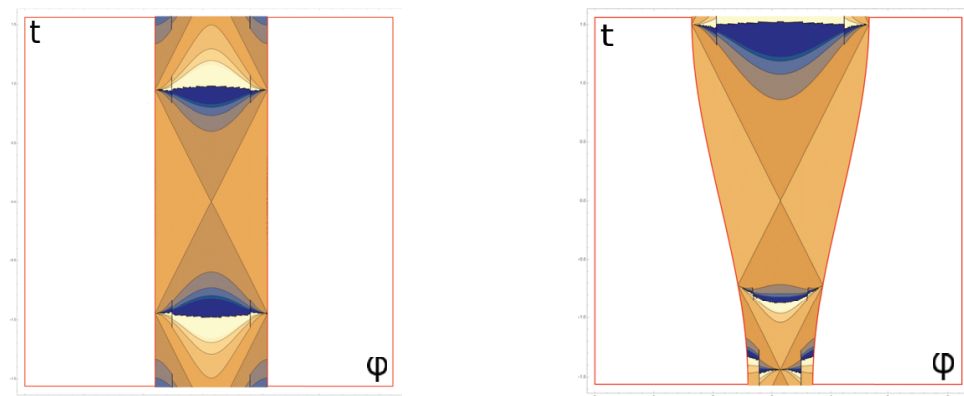

Figure 3. Density plot of inverse correlation function as a function of $G^{-1}(t, \varphi)$ is presented. In the both pictures non orbifold cases are presented. Parameter values are: $\xi=0, \alpha=4 \pi / 3+0.2$. In the left plot the massive particle is static. In the right plot the massive particle is moving. Parameter values are: $\xi=0.6, \alpha=4 \pi / 3+0.2$

\section{Acknowledgments}

This paper is based on a talk by 19th International Seminar on High Energy Physics "Quarks-2016" at Pushkin, Russia on 29 May - 4 July 2016. Author would like to thank the organizers of Quarks-2016 for the support. Author would like to thank Dmitrii Ageev, Irina Arefeva and Mikhail Khramtsov for useful discussions. The author is supported by the Russian Science Foundation (project 14-11-00687). 


\section{References}

[1] J. M. Maldacena, "The Large N limit of superconformal field theories and supergravity," Adv. Theor. Math. Phys. 2, 231-252 (1998), [hep-th/9711200].

[2] V. Balasubramanian and S. F. Ross, "Holographic particle detection," Phys. Rev. D 61, 044007 (2000) [hep-th/9906226].

[3] V. Balasubramanian et al., "Thermalization of the spectral function in strongly coupled two dimensional conformal field theories," JHEP 1304, 069 (2013) [arXiv:1212.6066 [hep-th]].

[4] I. Arefeva, A. Bagrov, P. Saterskog and K. Schalm, "Holographic dual of a time machine," arXiv:1508.04440 [hep-th].

[5] I. Y. Aref'eva and M. A. Khramtsov, "AdS/CFT prescription for angle-deficit space and winding geodesics," arXiv:1601.02008 [hep-th].

[6] S. S. Gubser, I. R. Klebanov, A. M. Polyakov, "Gauge theory correlators from noncritical string theory," Phys. Lett. B428, 105-114 (1998), [hep-th/9802109].

[7] E. Witten, "Anti-de Sitter space and holography," Adv. Theor. Math. Phys. 2, 253-291 (1998), [hep-th/9802150].

[8] S. Deser, R. Jackiw, and G. 't Hooft, "Three dimensional Einstein gravity: dynamics of flat space", Ann. Phys. 152 (1984) 220

[9] S. Deser and R. Jackiw, "Three-Dimensional Cosmological Gravity: Dynamics of Constant Curvature," Ann. Phys. 153 (1984) 405.

[10] D. S. Ageev, I. Y. Aref'eva and M. D. Tikhanovskaya, "Holographic Dual to Conical Defects: I. Moving Massive Particle,” Theoret. and Math. Phys., 188:1 (2016), 1038-1068 [arXiv:1512.03362 [hep-th]].

[11] I. Ya. Aref'eva, M. A. Khramtsov and M. D. Tikhanovskaya, "Holographic Dual to Conical Defects III: Improved Image Method," [arXiv:1604.08905 [hep-th]] 